\title{
Dynamic Strategies of Conflict Resolution on Human Perception of Equality within Multi-user Collaborative Virtual Environments
}

\author{
Aida Erfanian, Tao Zeng and Yaoping $\mathrm{Hu}$ \\ Department of Electrical and Computer Engineering \\ University of Calgary \\ Calgary, Alberta, CANADA \\ \{aerfania@ucalgary.ca; tzeng@ucalgary.ca; huy@ucalgary.ca\}
}

\begin{abstract}
Multi-user collaborative virtual environments (VEs) need strategies of conflict resolution to handle simultaneous interaction with shared objects. Current strategies are first-comefirst-serve (FCFS) and predefined static priority of each user. These strategies cannot provide each user with a perceived equal opportunity of interaction and often lead to perceived unfairness to abandon collaboration. To offer an equal opportunity, we created a dynamic priority (DP) strategy and compared the strategy with the FCFS strategy based upon subjective perception of multiple users. Visual or haptic (pertinent to the sense of touch) cues assisted each user to perceive his/her gaining of interaction. We observed that the DP strategy yielded significantly an equal opportunity of interaction. The haptic cue offered lower variations in perceiving the equality than the visual cue under the DP strategy. These observations imply a potential application of the DP strategy in a VE, where various experts require equal opportunities in collaboration.
\end{abstract}

Keywords- conflict resolution; multi-user collaborative virtual environments; dynamic priority strategy; human perception

\section{INTRODUCTION}

Computer-based virtual environments (VE) play an undeniably important role in intuitive and real-time user interaction. This could yield collaborative VEs to permit colocated and synergistic interaction of multiple users. Most current VEs offer one interactive device for co-located users, allowing one active user to control the device for collaboration along with multiple passive audiences [1]. Consequently, this type of VEs has a deficit of causing dissatisfaction among the users and preventing them from genuine collaboration [2].

To mitigate this deficit, each user could utilize an interactive device in a co-located collaborative VE facilitated with appropriate strategies for coordinating multi-user interaction. Such strategies are especially crucial to handle conflicting interactive commands, when multiple users attempt simultaneously to manipulate a shared object. Generally, these strategies fall into two categories: conflict avoidance and conflict resolution. On one hand, the strategies of conflict avoidance often restrict each user to interact with an object within a distinctive region and impose the rule of non- simultaneous interaction with an object in a specific shared region. The distinctive region is either statically pre-defined before commencing collaboration [3] [4] or dynamically changeable during undertaking collaboration [5]. When the conflict of interactive commands is inevitable in the shared region, the users should follow specific 'social protocols' - a set of behavioral rules imposed among the users - to handle the conflict [5] [6]. Thus, the users avoid issuing conflicting interactive commands as many as possible. Nevertheless, the 'social protocols' are in general useless due to the overhead of imposing the rules and cause dissatisfaction among the users [5]. Many studies have employed the strategies of conflict avoidance in applications of multi-touch tabletops and large tiled-displays [3] [4] [6] [7].

On another hand, the strategies of conflict resolution cope with the conflict of interactive commands by granting one of the users an exclusive access to a shared object. The exclusive access arises when the user has a pre-defined static priority (SP) higher than the other users [3]; or when the user issues an interactive command at the earliest instant among the users with the same priority. The latter is referred as first-come-first-serve (FCFS) and depends dynamically upon each user's agility of issuing an interactive command. Indeed, FCFS is the mostly common strategy to handle conflicting commands among the users with the same SP explicitly or implicitly. Nevertheless, the difference among the users' agilities could result in perceived 'suboptimal interaction' to impact negatively on the progress of collaboration [8].

Although many research groups have undertaken studies on strategies for coordinating multi-user interaction [2] [3] [9] [10] [11], few has assessed strategies of conflict resolution within a collaborative VE. Some researchers have theoretically proposed various strategies of conflict resolution [12]. With certain technical merits, all of these strategies neglect the consideration of human perception on collaboration. To our best knowledge, there have been lacking investigations on the users' perception of equality within a multi-user collaborative $\mathrm{VE}$, resulted from various strategies of conflict resolution.

This work is supported by the NSERC-SPG grant (STPGP/364847-2008) to Y. Hu. 
In practice, the demand for adequate strategies of conflict resolution becomes imperative in a multi-user collaborative VE, especially when each user expects to gain an equal opportunity of interaction with a shared object in collaboration. For example, one team of experts in various technical domains such as oil/gas reservoir engineers, drilling engineers, geologists and geophysicists - routinely solves a problem of their interest by working together to determine certain characteristics of a 3D geological dataset (a shared object). For such a collaborative endeavor, it is hence imperative to have an adequate strategy of conflict resolution that could effectively offer each user a perceived equal opportunity of interacting with the shared object to express his/her own idea. Importantly, this equality could allow valuable ideas to flourish, as a result of eliminating perceived inequality or "suboptimal interaction" [8]. Moreover, collaborative endeavors involving a team of experts usually require exploring a large quantity of 3D complex data. To facilitate the human understanding of the data, it is thus vital for a collaborative VE to represent the data in stereoscopic visualization for interaction [13].

To offer an equal opportunity of interaction, we proposed and implemented a strategy of conflict resolution called "dynamic priority" (DP) for a multi-user collaborative VE. At a time instant, the interactive priority of each user is dependable upon his/her history of interaction. To verify whether the users would subjectively perceive the theoretical equality of interaction offered by the DP strategy, we conducted an empirical study to compare the uses' perception of interaction under the DP strategy with that under the FCFS strategy - a dynamic strategy most commonly used in collaborative VEs. In a co-located 3D stereoscopic VE, we carried out the comparison under both visual cue (unique to each user) and haptic cue (pertinent to force feedback felt by the user's hand) to allow each user to recognize whether he/she has taken control of the interaction. In the VE, each user utilized an identical device, which is able to feed a force to the user's hand, for interacting with a shared object. We observed that human participants (as the users) perceived an equal opportunity of interaction under the DP strategy. Furthermore, the haptic cue offered significantly lower variations in perceiving the equality than the visual cue under the DP strategy.

We organize this paper as follows: Section II explains the implemented strategies of conflict resolution; Section III presents our co-located multi-user collaborative VE; Section IV describes our empirical study with its method, observed results and discussion; and Section V concludes the observation with future work.

\section{StRAtegies OF CONFLict Resolution}

For multiple users issuing their interactive commands simultaneously, we considered two dynamic strategies of conflict resolution: FCFS and DP.

\section{A. FCFS strategy}

Although being easy to implement and mostly common in collaborative VEs, FCFS is known as an unfair strategy. Basically, FCFS promotes "winner-takes-all” for the most agile user to take control of interaction with a shared object and thus to prevent the less agile users from taking part in collaboration. An improved version of the FCFS strategy, called the SP strategy, assigns each user a priority based upon his/her predefined role in the interaction [3]. For example, the user who functions as a presenter has a higher priority than other users who play a role of audience. These roles are static and, in turn, unchangeable during the course of collaboration. In the case of multiple users issuing interactive commands simultaneously on a shared object (that is, a conflict of commands occurs), the user with a higher priority wins the interaction with the shared object. If the multiple users have the same SP, the agilest one gains the interaction based on FCFS. For our study, we considered that all users have the same priority and thus implemented the FCFS strategy in the same way as that for the users of audience in the SP strategy.

\section{B. DP strategy}

At the beginning of the collaboration, we assumed that each user has the same initial priority (as a probability). To give each user an equal opportunity of interacting with a shared object during the course of collaboration, we proposed a DP strategy by updating dynamically the priority (the probability) of each user according to his/her history of interaction. For example, if there are a total of $n$ users and $m$ trials (attempts) in collaboration, the priority of the $i$-th user at the $k$-th trial (attempt) is formulated as follows:

$$
\begin{aligned}
& \text { priority }_{i, k}=\frac{\text { priorityNumerator }_{i, k}}{\text { priorityDenominator }_{i, k}} \\
& =\frac{\text { priorityNumerator }_{i, k-1}+f(i, k-1)}{(n-1) k+1} ; \\
& i \in\{1,2,3, \ldots, n\}, \quad k \in\{1,2,3, \ldots, m\} \text {; } \\
& f(i, k-1)= \begin{cases}1, & c_{1} \\
0, & c_{2}\end{cases} \\
& c_{1} \text { : the } i \text {-th user could not take the control of ( } k \text {-1)-th trial; } \\
& c_{2} \text { : the i-th user took the control of the (k-1)-th trial. }
\end{aligned}
$$

For the $i$-th user, the numerator of the current $k$-th trial is calculated based on the numerator of the previous $(k-1)$-th trial and a participation function $f(i, k-1)$, as indicated in Eq. (1). The function $f(i, k-1)$ represents whether the $i$-th user gains the control of the interaction in the previous $(k-1)$-th trial. If the user has this control, the numerator of the current $k$-th trial remains unchanged; otherwise, this numerator is incremented by 1 . In this way, the fractional numerator of the users who could not take part the interaction in the previous $(k-1)$-th trial is increased for the current $k$-th trial, while keeping the numerator of the user who has undertook the interaction in the previous $(k-1)$-th trial unchanged for the current $k$-th trial. As well, the denominator is increased for the current $k$-th trial to maintain the summation of all priorities (probabilities), priority $_{i, k}$, within the bound of one. 


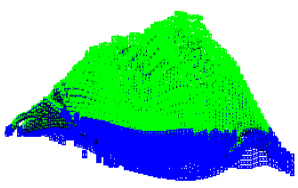

(a)

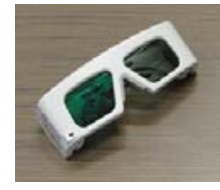

(b)

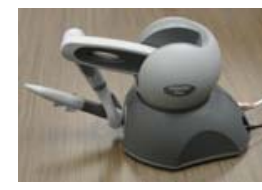

(c)
Figure 1: A collaborative VE with: a) a geological dataset; b) a pair of active 3D stereoscopic goggles and c) a Omni haptic device.

To grant all users an equal opportunity of interaction, we considered the visuomotor response of each user in addition to his/her priority determined by Eq. (1). This consideration derived from the fact that all users have different characteristics of motor responses to identical visual stimuli; because of their individual ages, physical fitness and abilities of concentration. Consequently, all users would possess different time lengths for the identical visuomotor response. For this reason, we defined a time interval $x$-interval. All interactive commands issued by the users within the $x$-interval were considered to occur simultaneously. When multiple users attempted simultaneously (within the $x$-interval) to interact with a shared object, the user with the highest priority determined by Eq. (1) undertook the interaction. Once the interaction was completed, the priorities of all users were updated by using Eq. (1) for the next trial (or attempt). For each user, this update took account his/her history of success in gaining the interaction: the priority of all users, except the one who had just completed the interaction, would be relationally incremented to enhance their chance of gaining interaction in the next trial. This increment in the priority was fractional to keep the sum of all users' priorities equal to one.

\section{A Multi-User Collaborative VE}

To assess the effect of both FCFS and DP strategies on the users' perception of equality in interaction, we developed a collaborative VE for multiple co-located users.

\section{A. Equipment}

The VE displayed a geological dataset, as shown in Fig. 1(a), on a wall-sized screen for all users to share for interaction. Each user utilized a pair of stereoscopic goggles, as illustrated in Fig. 1(b), to view the shared object in a 3D stereoscopic display. For each user, we provided a PHANToM ${ }^{\circledR}$ Omni device, as depicted in Fig. 1(c), as a haptic interface to interact with the shared object. Due to the availability of the Omni devices, we connected three such devices (for three users) to a graphic computer with a 64-bit Windows ${ }^{\circledR} 7$ operating system, a $2.53 \mathrm{GHz}$ (dual quad core processors) Intel ${ }^{\circledR}$ Xeon ${ }^{\circledR} \mathrm{CPU}$, a 4GB RAM and a Quadro FX 4800 NVidia ${ }^{\circledR}$ graphics card. By using $\mathrm{C}++$, OpenGL and OpenHaptics, we implemented our software application for interacting with the shared object by a user via an Omni device at one time instant; although all users could issue their interactive commands simultaneously. This implementation used a multi-threading software architecture: one visual thread for displaying objects and another haptic thread for interacting with the shared object via an Omni device - even though multiple Omni devices issued interactive

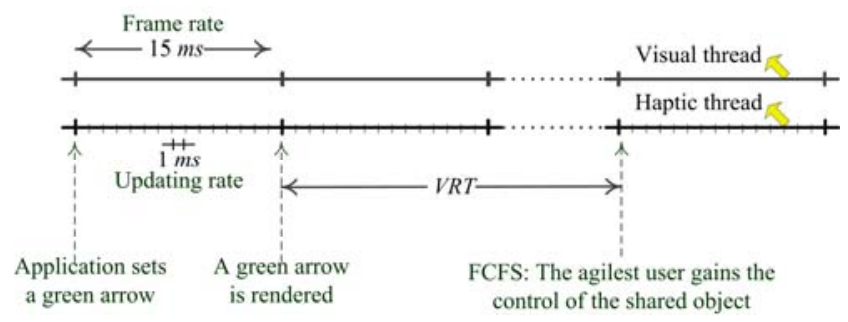

Figure 2: The timing relationship among the concepts of the FCFS strategy.

commands. To provide a 3D stereoscopic view, we utilized the center screen of a computer aided virtual environment (CAVE) with 4 wall-sized screens (left, center, right and floor). For all users, either the FCFS or DP strategy governed conflict resolution for interacting with the shared object.

\section{B. Implementation of the FCFS strategy}

For real-time user interaction, we considered the following concepts in implementing the FCFS strategy:

- $\quad$ Frame rate of the OpenGL rendering;

- Updating rate of the OpenHaptics scheduler;

- Visuomotor response time (VRT) of the humans.

- Interrupt latency: the time between the 'button down' event of an Omni device and the algorithm of the FCFS strategy received this event. This latency was so small that we lumped it into VRT.

Fig. 2 depicts the timing relationship of these concepts. In our application, the frame rate of the OpenGL rendering and the updating rate of the OpenHaptics scheduler were averagely measured as about $66 \mathrm{~Hz}$ (15 ms) and $1 \mathrm{kHz}(1 \mathrm{~ms})$, respectively. These measurements assisted us to estimate the time length needed to indicate the beginning of a trial (by rendering a visual arrow in green) without the users' conscious notice of this time length. We applied the VRT of the users as $200 \mathrm{~ms}$, the upper boundary of the VRT according to neuroscience literature [14]. Thus, each user would press the dark grey button of his/her Omni device about $215 \mathrm{~ms}$ after the application set the beginning of a trial.

Our algorithm implementing the FCFS strategy used a queue to hold the interactive commands of all users - their pressing the dark button of their Omni devices. The user whose interactive command was at the first spot of the queue would be the one to win the interaction with the shared object during the trial. The haptic thread handled this exclusive access of the shared object, because all haptic devices were under the control of the OpenHaptics scheduler. When a trial ended, the next trial began with an empty queue.

\section{Implementation of the DP strategy}

In the DP strategy, each user had a priority to imply his/her probability of gaining control of the shared object in a trial. As well, all interactive commands issued by the users within the $x$ interval would be considered as occurring simultaneously. That is, the $x$-interval eased the difference of agility among the users of pressing the dark grey button of their Omni devices. The algorithm implementing the DP strategy applied a queue to 


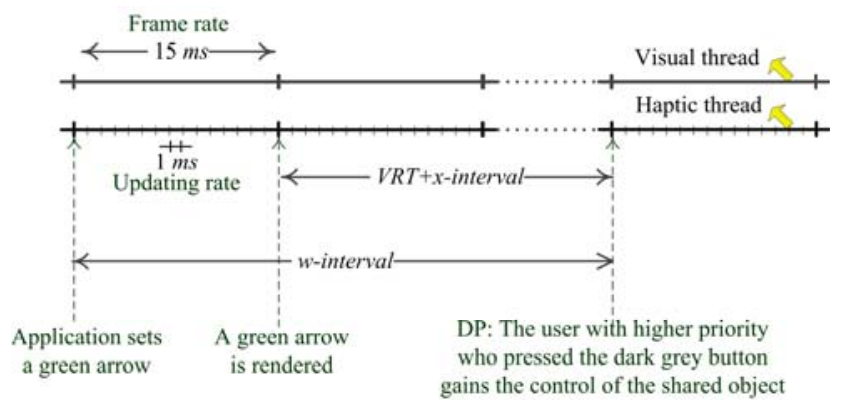

Figure 3: The timing relationship of all concepts in the DP Strategy.

hold the interactive commands issued by all users. We considered a w-interval (whole-interval) between the beginning of a trial by setting a visual arrow in green and the time instant of granting the user with the highest priority (plus pressed the dark grey button of his/her Omni device) to gain the control of the interaction with the shared object. For implementing the DP strategy, the frame rate of the OpenGL rendering, the updating rate of the OpenHaptics scheduler, VRT and interrupt latency were the same as those for implementing the FCFS strategy. Fig. 3 illustrates the timing relationship of these and the following concepts for assessing the amount of the w-interval:

- Rendering latency (RL): the time elapsed between setting the green color to an arrow in the VE to rendering the green-colored arrow. This delay was one frame of the OpenGL rendering ( $15 \mathrm{~ms}$ ).

- The $x$-interval: an interval should be larger than one VRT considering the human visuomotor constraints and various latencies described above. Through several pilot studies, we found that the maximally allowable $x$-interval was about $500 \mathrm{~ms}$ in our VE. Longer than $500 \mathrm{~ms}$, the users reported a noticeable pause between issuing interactive commands and initiating the shared object. For our empirical study described below, we set the $x$-interval to be $285 \mathrm{~ms}$.

Consequently, we defined Eq. (2) to estimate the w-interval

$$
w_{-} \text {interval }=R L+V R T+x_{-} \text {interval }
$$

and set the w-interval to be $500 \mathrm{~ms}$.

\section{Visual and haptic cues}

We used two distinct types of feedback to facilitate the users' recognition of their control of the shared object in each trial: visual and haptic cues.

The type of the visual cue was used in the trials that visual feedback was the only mean for each user to recognize his/her control of the shared object. Although each user employed an Omni device to issue his/her interactive command, no haptic feedback (force) was rendered to the user' hand in these trials. We assigned a unique shape of the cursor to an Omni device: the sphere-, cube- and torus-shaped cursor corresponding to the first, second and third Omni device in our VE, respectively. When one user took control of the shared object, the cursor corresponding to the Omni device in use would appear on the

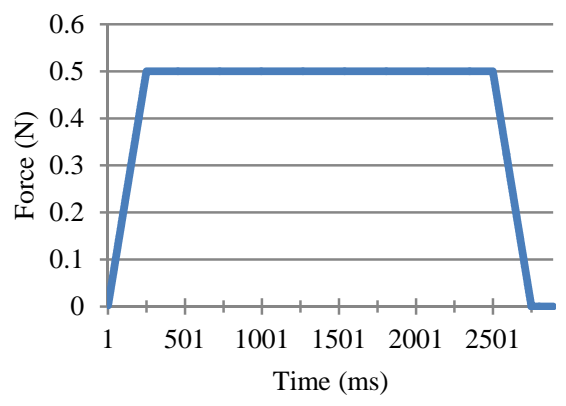

Figure 4: The profile of the haptic cue.

display screen. All users could view this cursor in the same way as they view the shared object.

The type of the haptic cue was employed in the trials that haptic feedback functioned as the only mean for each user to recognize his/her control of the shared object. No particular shaped cursor was visibly provided in these trials. We rendered a trapezoidal force to the user's hand via his/her Omni device, when the user took the control of the shared object in a trial. For about $2.25 \mathrm{~s}$, the user felt a resistant force $(0.5 \mathrm{~N})$ against the movement of the share object. The trapezoidal force ensured a gradual rise and fall of the force to avoid any jolt on the stylus of the Omni device, because the jolt could cause discomfort to the user. Fig. 4 illustrates the shape and magnitude of the force for feedback. The maximum amplitude of the continuous force was $0.5 \mathrm{~N}$ to balance between the feel of the force comfortably by the user and the noise-free operation of the device.

\section{EMPIRICAL STUDY}

In the VE described above, we conducted an empirical study using human participants as the users to examine whether the users could perceive equal opportunities of interaction under the DP strategy, compared to the FCFS strategy. The study had an ethics clearance following the Canadian Tri-Council guidelines.

\section{A. Participants}

We recruited a total of 30 participants (16 males and 14 females, with the average age of $25.67 \pm 3.78$ years), who were all over 18 years old and naïve to the purpose of the study. They had normal to corrected-to-normal vision with a stereo acuity of at least $40^{\circ}$ arc (determined using the Randot Stereo-test) and no difficulty recognizing colors determined using the Ishihara color-blindness test. They were all right-handed and had no impairment for holding a stylus, tested using a modified version of the Edinburgh Handedness Inventory. These participants formed 10 groups; three participants (the users) of each group undertook co-located multi-user collaboration within our VE. The sample size of the 10 groups was larger than the minimal sample size (8) calcuated by using the Lehr's formular [15] for a within-subject-design and repeated-measure comparison, as decribed below.

\section{B. Procedure}

As shown in Fig. 5(a), three seats were placed in front of the center screen of the CAVE at a distance of about $650 \mathrm{~cm}$ from the screen. The seat of the center-seated participant was aligned 

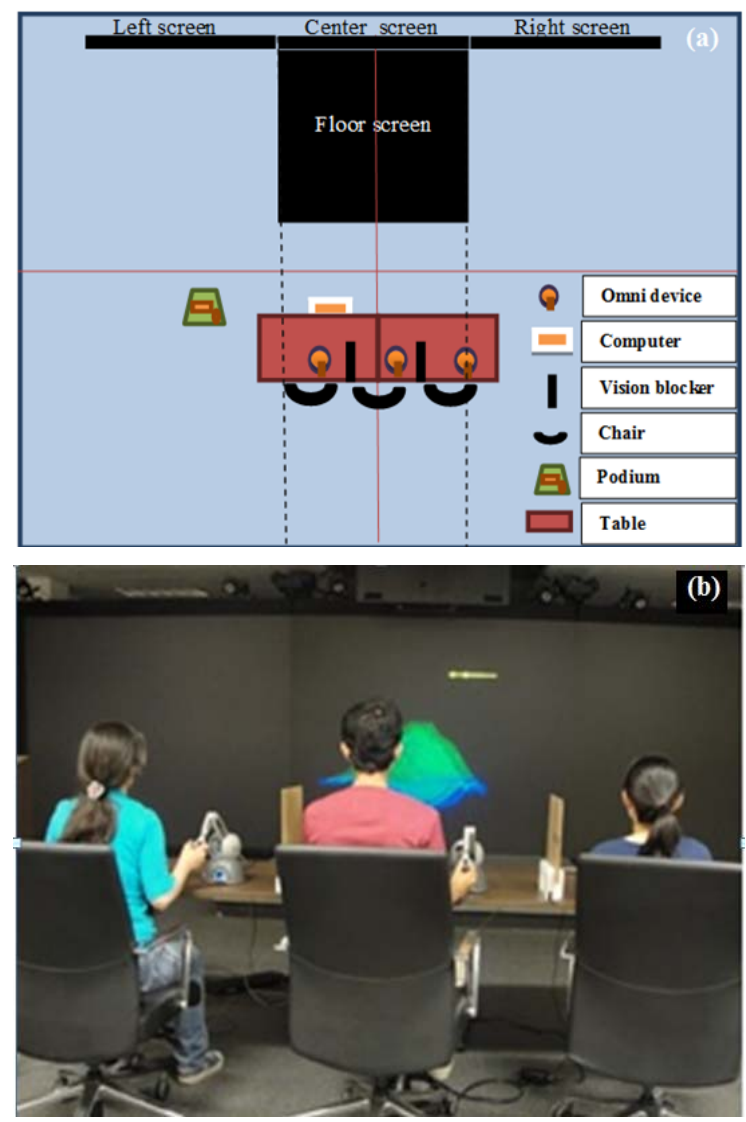

Figure 5: The set-up of the empirical study. a). Room layout for conducting the study. b). Example of three participants interacting with the shared object in our VE.

to the middle of the center screen. The seats of other participants were placed in an equal distance from the centered seat. The graphic computer described earlier was on the floor and under the table, so that the computer did not hinder any user to view the $3 \mathrm{D}$ stereoscopic display of the center screen. We placed the keyboard and mouse on the podium to permit one researcher to monitor the study and to control the software application for the study. Another researcher sat behind the participants during the study to monitor their interaction without any interference.

As shown in Fig. 5(b), each Omni device was aligned with the right arm of each seat to ensure that a right-handed user could utilize the device with his/her right (dominant) hand. Two flat obstacles as vision blockers were placed between two users. The vision blockers prevented distractions from a glimpse of the other user's hand. During the experiment, each user and researcher wore a pair of active goggles to view the display on the center screen in 3D stereoscopy.

The experiment was a within-subject-design comparison each group of the users underwent the same procedure of two sessions: a visual session of using the visual cue and a haptic session providing the haptic cue. Each session had 3 blocks including one practice block and two testing blocks. The practice block allowed the users to familiar with the cues provided and the actions that they needed to take. After completing the practice block, the users underwent two testing blocks governed under the FCFS strategy and the DP strategy, respectively. Thus, the two sessions yielded totally 4 testing blocks as 4 different testing conditions: FCFS+V (the FCFS strategy with visual cue); FCFS $+\mathrm{H}$ (the FCFS strategy with haptic cue); $\mathrm{DP}+\mathrm{V}$ (the DP strategy with visual cue) and $\mathrm{DP}+\mathrm{H}$ (the DP strategy with haptic cue).

In each trial of a block, we presented a yellow arrow pointing to a direction and the shared object of a geological dataset, as shown in Fig. 5(b). All users were instructed to press the dark grey button of their Omni devices, as soon as seeing the arrow turned into green (signaling the beginning of a trial), and to translate the object along the arrow-pointed direction simultaneously. However, only one user employed his/her Omni device to move the shared object actually. One unique visual or haptic cue was presented to the user indicating his/her success of interacting with the shared object, although all users viewed the movement of the object and the visual cue (except the haptic cue). Each trial lasted about $10 \mathrm{~s}$ and then the green arrow turned into yellow to end the trial.

In each block, there were a total of 6 various arrow-pointed directions, corresponding to all positive and negative axial directions in a Cartesian coordinate system. We replicated each arrow-pointed directions 5 times, resulting a total of 30 trials for each block. At the end of each block (except the 2nd practice block), we distributed an identical questionnaire to each user to filled out. The two sessions lasted together about one hour, including short breaks from 3 to 5 minutes between two blocks. The 2 sessions and 2 testing blocks of each session were counterbalanced for all groups of the users.

\section{Data collection and analyses}

During the testing blocks, we used two methods to collect data. One method logged automatically via our application the user who gained the control of the shared object in each trial (governed by the FCFS or DP strategy), as well the historical priorities of all users in each trial governed by the DP strategy. This log created objective data about the actual interaction with the shared object for all users in each testing block.

Another method collected answers from the distributed identical questionnaires. This collection yielded subjective data about each user's perception of his/her opportunity in interacting with the shared object in each testing block. Each questionnaire consisted of two parts: The first part asked each user to mark a vertical line on a horizontal bar (bounded from $0 \%$ to $100 \%$ ) to indicate his/her perception (that arose in his/her mind) of gaining the interaction in a testing block. The second part was based on the valid NASA Task Load Index [16] to assess the workload perceived by each user in a testing block. We converted these answers into numeric for analyses.

We examined the objective data to verify whether there was any misbehaver (as an outlier) among the users of each group. From these data, we then calculated the mean and standard deviation of the percentages of the interaction with the shared object by all users in each group. Although the means for all testing blocks should be the similar (about 33.3\%) considering 
the three users in each group, the standard deviations for these blocks deserved our attention of investigation. The standard deviations for the blocks $\mathrm{DP}+\mathrm{V}$ and $\mathrm{DP}+\mathrm{H}$ should be zero due to the theoretical definition of the DP, priority $y_{i, k}$, in Eq. (1); whereas the standard deviations for the blocks FCFS+V and $\mathrm{FCF}+\mathrm{H}$ should be non-zero, indicating that some users were more agile than the others. That is, these means and standard deviations offered references for analyzing the subjective data.

Using these references, we expected the analysis of the subjective data to reveal an insignificant difference among the means of the perceived percentages of the interaction for all testing blocks, indicating that there was no misbehaver in the users' response to the questionnaires. To investigate the users' perception of equal opportunity in the interaction, we thus focused on the standard deviation of the perceived percentages of the interaction for all testing blocks. We conducted these analyses of the subjective data in the following steps: a) precondition tests, b) analysis based on the standard deviations, c) analyses based on the highest and lowest bounds of the perceived percentages, and d) analysis of the workload.

For the step a), the precondition tests were normality tests (normal probability density function [17]) to ensure that the distribution of the data for each testing block was suitable for applying the statistical method of analysis of variances (ANOVA)[18], and the average percentages of the perceived interaction for all testing blocks were not significantly different from each other. For the latter, we calculated the average percentage for each testing block as follows:

$$
a=\frac{\sum_{j=1}^{10}\left(\sum_{i=1}^{3} p_{i, j} / 3\right)}{10}
$$

where $a$ is the average percentage of the perceived interaction among the users of all groups and $p_{i, j}$ is the percentage of the interaction for the $i$-th user in the $j$-th group. Using one-way ANOVA (within-subject-design and repeated-measure), we compared the average percentages among all testing blocks $(\mathrm{FCFS}+\mathrm{V}, \mathrm{FCFS}+\mathrm{H}, \mathrm{DP}+\mathrm{V}$ and $\mathrm{DP}+\mathrm{H})$.

In the step b), we analyzed the standard deviations of the perceived interaction for investigating the users' perception of equality in the interaction governed by the FCFS strategy and the DP strategy, respectively. We executed a two-way ANOVA (within-subject-design and repeated-measure) to examine the effect of both interactive strategies (the FCFS and DP strategies as one way of two variables) and feedback cues (visual and haptic cues as another way of two factors) on the users' perception of equality in the interaction. When the two-way ANOVA revealed a statistical significance on interactive strategies or feedback cues, we don't need to employ one-way ANOVA to further investigate this significance because of each way has only two factors. Finally, we performed analyses of two-tail paired $t$-test to indicate whether there is a difference between the standard deviations of the perceived interaction for each pair of the testing blocks.

The step b) compared only the mean of the standard deviations of the perceived interaction. To ensure a full picture

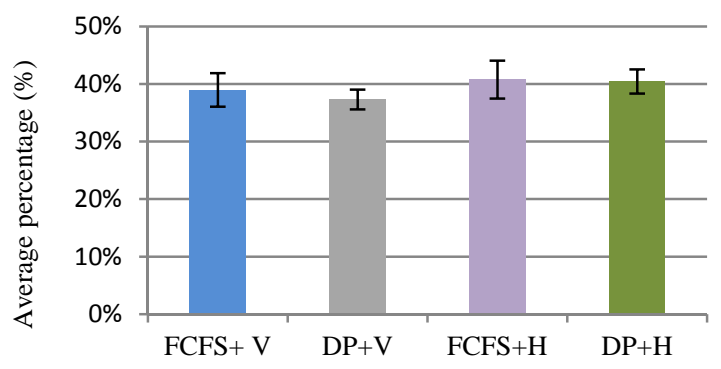

Figure 6: Average perceived percentage of the interaction with the shared object among all groups. [Error bars represent standard errors].

of the users' perception of equality in the interaction, we used ANOVA analyses to examine whether there were significant differences at the highest and lowest bounds of the perceived percentages of the interaction among all testing blocks in the steps c). We employed one-way ANOVA to analyze the means of overall workload among all testing blocks in the final step d).

\section{Results}

The analyses of the objective data revealed that, for each testing block, the total percentage of the interaction among three users of each group was $100.0 \%$. This revelation confirms that there was no misbehaver among the users of each group. The means of these percentages for each group were the same at about $33.3 \%$ for all testing blocks. For all groups, the standard deviations of the percentages were indeed zero for the blocks $\mathrm{DP}+\mathrm{V}$ and $\mathrm{DP}+\mathrm{H}$; and non-zero for the blocks FCFS $+\mathrm{V}$ and FCFS $+H$. These confirm the validity of the users' behavior for analyzing the subjective data.

Precondition tests: Normality tests verified that the distribution of the subjective data were normally distributed for all testing blocks. Using Eq. (3), we calculated the average perceived percentages of the interaction for each block. As depicted in Fig. 6, these averages were from $35.0 \%$ to $40.0 \%$-being below $50.0 \%$ and close enough to the theoretical $33.3 \%$. One-way ANOVA on these averages indicated that these percentages of the interaction had no significant difference among these blocks $[F(3,27)=0.38 ; p>0.05]$. The observation indicates that the standard deviations of these percentages would be a next parameter to compare for inquiring the perceived equality in interaction.

Analysis based on standard deviations: Fig. 7 illustrates the mean standard deviations under the FCFS and DP strategies (interactive strategies) for both visual and haptic cues (feedback cues), respectively. The FCFS strategy had larger mean standard deviations than the DP strategy, as illustrated in Fig. 7. Two-way ANOVA (interactive strategies $x$ feedback cues) revealed a significant difference for interactive strategies [ $F(1$, $18)=9.90 ; p<0.01]$ and no differentiability for feedback cues $[F(1,18)=1.40 ; p>0.05]$. No interaction existed between interactive strategies and feedback cues $[F(1,36)=0.37 ; p>$ 0.05]. To further investigate individual effect of feedback cues and of interactive strategies, we undertook the analyses of twotail paired $t$-test among all testing blocks. As shown in Table I, 


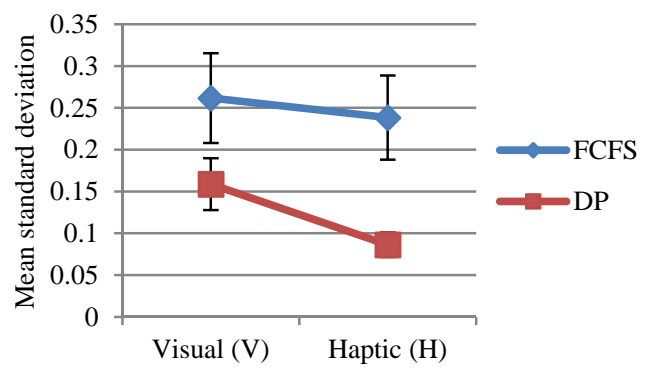

Figure 7: The mean standard deviations for the FCFS and DP strategies. [Error bars represent standard errors].

TABLE I. Paired $t$-test analyses among all testing blocks.

\begin{tabular}{|c|c|c|}
\hline Comparison & Two-tail paired $t$-test & $\begin{array}{c}\text { Significant } \\
\text { difference }\end{array}$ \\
\hline FCFS+V vs. DP+V & $t(9)=1.49 ; p>0.05$ & No \\
\hline FCFS+H vs. $\mathrm{DP}+\mathrm{H}$ & $t(9)=2.66 ; p<0.05$ & Yes \\
\hline FCFS+V vs. FCFS+H & $t(9)=0.75 ; p>0.05$ & No \\
\hline DP+V vs. DP+H & $t(9)=2.50 ; p<0.05$ & Yes \\
\hline
\end{tabular}

the visual cue did not offer differentiable effect between the FCFS and DP strategies, whereas the haptic cue did. Under the FCFS strategy, the effect of visual and haptic cues were not differentiable. In contrast, the same feedback cues offered a significant difference under the DP strategy.

Analysis based on the highest and lowest bounds of the perceived percentages: A two-way ANOVA (interactive strategies $\mathrm{X}$ feedback cues) on the highest bound (mean + standard deviation) of the perceived percentages showed that there was a significant difference for interactive strategies [ $F(1$, 18) $=6.63 ; p<0.05]$ and no differentiability for feedback cues $[F(1,18)=0.99 ; p>0.05]$. No interaction existed between interactive strategies and feedback cues $[F(1,36)=0.12 ; p>$ $0.05]$. The average highest bound of the perceived percentages (68.2\%) under the FCFS strategy was much greater than its counterpart (51.8\%) under the DP strategy.

Same analyses on the lowest bound of the perceived percentages (mean - standard deviation) revealed the similar observation: significant difference for interactive strategies $[F$ $(1,18)=5.42 ; p<0.05]$ and no differentiability for feedback cues $[F(1,18)=3.36 ; p>0.05]$ and no interaction $[F(1,36)=$ $0.15 ; p>0.05]$. As well, the difference of the average lowest bound between the FCFS strategy (20.4\%) and DP strategy (27.5\%) was much smaller than the difference of the average highest bound between these strategies.

Analysis of workload: One-way ANOVA showed that there was no significant difference of overall workload among all testing blocks $[F(3,27)=0.32 ; p>0.05]$, as depicted in Fig. 8 . This reveals that the DP strategy had similar overall workload as that of the FCFS strategy.

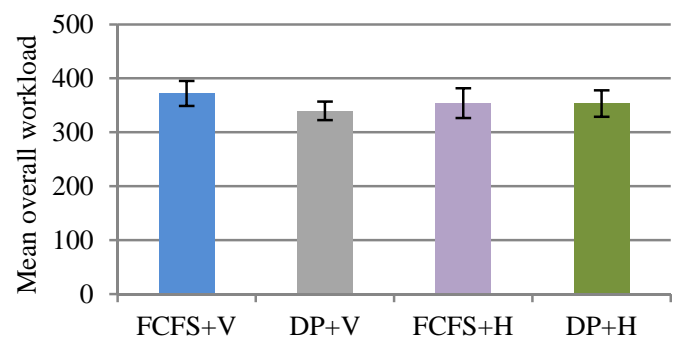

Figure 8: Overall workload under all testing blocks. [Error bars represent standard errors].

\section{E. Discussion}

Although the means of the perceived percentages of the interaction were similar under the FCFS and DP strategies, we observed that the standard deviation of these perceived percentages under the FCFS strategy was statistically larger than that under the DP strategy. That is, the FCFS strategy yielded a wider spread of the perceived opportunity of the interaction than the DP strategy, especially when the haptic cue was in use. This illustrates that the human users perceived significantly an equality of interaction under the DP strategy, evident by the much narrowed spread of the perceived percentages of the interaction, as depicted in Fig. 7.

Interestingly, the visual and haptic cues appeared to have no differentiable effect on the equality, disagreeing with the facilitation of haptic cues in user interaction found in literature [19]. Two aspects might contribute to this disagreement. In one aspect, our magnitude of the force $(0.5 \mathrm{~N})$ is at the threshold of the human perception of force. Below this threshold, the resolution of human perception of force deteriorates [20]. In another aspect, there is little literature about the role of haptic cue on the interactive strategy of conflict resolution. Nevertheless, the comparison of the standard deviations between the blocks $\mathrm{DP}+\mathrm{V}$ and $\mathrm{DP}+\mathrm{H}$ indicate that the haptic cue offered significantly lower variations in perceiving the equality than the visual cue under the DP strategy. Apparently, the DP strategy is suitable and intuitive for the users with the haptic cue than with the visual cue. No similar observation was noticeable under the FCFS strategy. Moreover, the perceived equal opportunity of interaction under the DP strategy did not increase the overall workload, compared to the FCFS strategy.

The FCFS strategy allows the agilest user to gain the control of interaction within a multi-user collaborative $\mathrm{VE}$ and leaves the slow users deprived of interaction. In contrast, the DP strategy offers an equal opportunity of interaction for all users by updating their priority according their history of interaction, No matter how agile the users would be, their interactive commands would be considered as simultaneous as long as they issue their interactive commands within a specific $x$-interval. The length of this $x$-interval is comparable to the human VRT, so the users would not notice a gap of waiting to hinder their interaction. Consequently, the users perceived much small variations in interaction under the DP strategy. 
We derived the DP strategy from a SP strategy [3]. The SP strategy resolves conflicting interactive commands by using a pre-defined and non-changeable priority corresponding to a pre-assigned interactive role of each user, assuming that each user has a unique role. In contrast, the DP strategy keeps the interactive priority of each user in equal by rebalancing the priority dynamically according to the historical interaction of each user. Theoretically, the SP strategy yields an unequal number of interaction for all users, whereas the DP strategy offers an equal number of interaction to these users. The users notice consciously this equal number of interaction, evident by the narrowed variation of the perceived percentages.

\section{CONCLUSIONS}

We proposed the DP strategy of conflict resolution and observed that, within a multi-user collaborative VE, this strategy provided a perceived equality in interaction and the haptic cue facilitated this perception. These observations imply a potential application of the DP strategy in the VE, where various experts require equal opportunities in genuine collaboration. Further work is to verify the effectiveness of the DP strategy for such an application.

\section{REFERENCES}

[1] V. Bayon, G. Griffiths, and J. R. Wilson, "Multiple decoupled interaction: An interaction design approach for groupware interaction in co-located virtual environments," Int'l J. Human-Computer Studies, vol. 64, no. 3, pp. 192206, 2006.

[2] A. Simon and C. Stern, "Coordination policies for colocated collaborative travel," Proc. Virtual Reality Conf., pp. 285-286, Reno, Nevada, USA, Mar. 2008.

[3] Y. Lou, W. Wu, and H. Zhang, "Magic input: A multi-user interaction system for SAGE based large tiled-display environment," Proc. ICMEW'12, pp. 157-162, Melbourne, Australia, Jul. 2012.

[4] M. A. Nacenta, D. Pinelle, C. Gutwin, and R. Mandryk, "Individual and group support in tabletop interaction techniques," Tabletops - Horizontal Interactive Displays, Human-Computer Interaction Series, pp. 303-333, 2010.

[5] M. Fetter, T. Gross, and M. Hucke, "Supporting social protocols in tabletop interaction through visual cues," Human-Computer Interaction - INTERACT 2011, part III, LNCS 6948, pp. 435-442, 2011.

[6] E. Tse, S. Greenberg, C. Shen, C. Forlines, and R. Kodama, "Exploring true multi-user multimodal interaction over a digital table," Proc. 7th ACM Conf. on Designing Interactive Systems, pp. 109-118, Cape Town, South Africa, Feb. 2008.

[7] D. Pinelle, M. Barjawi, M. Nacenta, and R. Mandryk, "An evaluation of coordination techniques for protecting objects and territories in tabletop groupware," Proc. SIGCHI Conf. on Human Factors in Computing Systems, pp. 2129-2138, Boston, MA, USA, Apr. 2009.
[8] Y. Rogers, Y. Lim, W. R. Hazlewood, and P. Marshall, "Equal opportunities: Do shareable interfaces promote more group participation than single user displays?," Human-Computer Interaction, Special Issue: Ubiquitous Multi-Display Environ, vol. 24, no. 1-2, pp. 79-116, 2009.

[9] D. Pinelle, T. Stach, M. A. Nacenta, D. Stuckel, and C. Gutwin, "Tabletop coordination: The effect of interaction techniques on group activities," Video Proc. CSCW 2006, Banff, Alberta, Canada, Nov. 2006.

[10] J. Stewart, B. B. Bederson, and A. Druin, "Single display groupware: a model for co-present collaboration," Proc. SIGCHI Conf. on Human Factors in Computing Systems, pp. 286-293, Pittsburgh, PA, USA, May. 1999.

[11] S. Otmane, N. Ouramdane-Djerah, and M. Mallem, "Towards a collaborative 3D interaction model for cooperative design in virtual environments," Proc. 11th Int'l Conf. on Computer Supported Cooperative Work in Design, pp. 198 - 203, Melbourne, Australia, Apr. 2007.

[12] M. R. Morris, K. Ryall, C. Shen, C. Forlines, and F. Vernier, "Beyond social protocols: Multi-user coordination policies for co-located groupware," Proc. ACM Conf. on Computer Supported Cooperative Work, pp. 262-265, Chicago, Illinois, USA, Nov. 2004.

[13] Satellite Imaging Corporation. Oil and gas exploration and production. [Online].

"http://www.satimagingcorp.com/svc/exploration.html"

[14] A. T. Welford, "Choice reaction time: Basic concepts”, Reaction Times, A. T. Welford, Ed, pp. 73-128, 1980.

[15] G. Van Belle, "Statistical rules of thumb”, WileyInterscience, 2002.

[16] Human Performance Research Group, NASA Ames Research Center. NASA TLX: Task Load Index. [Online]. "http://humansystems.arc.nasa.gov/groups/TLX/ paperpencil.html"

[17] M. Evans, N. Hastings and B. Peacock, Statistical Distributions, 2nd ed., Wiley, 1993.

[18] K. Hinkelmann, and O. Kempthorne, Design and Analysis of Experiments, New York: Wiley, 2008.

[19] A. Widmer, Y. Hu, "Effects of the alignment between a haptic device and visual display on the perception of object softness," IEEE Trans. SMC - Part A: Systems and Humans, vol.40, no.6, pp.1146 - 1155, Nov. 2010.

[20] H. K. Kim, D. W. Rattner, and M. A. Srinivasan, "Virtualreality-based laparoscopic surgical training: The role of simulation fidelity in haptic feedback," Computer Aided Surg., vol. 9, no. 5, pp. 227- 234, 2004. 